\title{
POSITIONING FOR PEOPLE BEHIND BARRIERS IN REAL TIME WITH UWB PULSE SENSING
}

\author{
Sergey Shipilov ${ }^{1,}$, Rail Satarov, Ivan Fedyanin, Evgeny Balzovsky, VladimirYakubov \\ National Research Tomsk State University, 634050, Tomsk, Russia
}

\begin{abstract}
This paper features the technology for identifying the location of real people behind the barriers in real time using the UWB sensing. The opportunity to obtain the radar images of moving and not moving people was presented in this paper. The oscillation rate of a human chest can be defined by the continuous recording the not moving person. The point of this technique is in calculation of the differential signal in successive periods of time. The range of application to be extended considering the particular conditions. The research results can be used in intelligence service as well as for searching people trapped under the rubble.
\end{abstract}

\section{Introduction}

Terrorism and counter terrorism policy as well as its impact on everyday life are of high relevance nowadays as there is always a high chance of hostage-taking or destruction of the engineering facilities. In both cases, every second counts for human lives. Therefore, the equipment for remote accurate detecting people in real time behind the barriers are very important.

The use of X-rays is preferred for that purpose because of high resolution and penetration. However, its hazard for living beings and the one-side access to the test object prevent X-rays from the application in the searching and detecting people behind barriers. Therefore, the radiowave tomography techniques are the most reasonable choice.

The ultra-wideband (UWB) radiation provides the high longitudinal spatial resolution, which is particularly important to detect hidden objects behind the dielectric barriers. The radiation of this type provides the sufficient penetration into a barrier to create a radiotomogram of the test area. This process is based on tomography of multi-angle remote measurements of scattered radio wave radiation. Radiowave tomography is anyway based on ray-focusing effect, which enables inverse transformation of wave projections of test objects and propagation medium. It appears that almost all methods which are available or just in progress now, may be considered under this aspect. The multiple effects (scattering and diffraction) of interacting the wave fields with propagation medium inhomogeneities can be considerably reduced by using of spatiotemporal radiation focusing.

The developed algorithms of creating the tomogram should be resistant to noise and interference, which significantly impact the received signal in the one-side sounding of the objects behind the dielectric barriers[1,2]. The developed algorithms should also provide

\footnotetext{
${ }^{1}$ Corresponding author: s.shipilov@gmail.com
} 
the adequate operating speed when the requirements to the measuring and recording equipment as well as to the computer hardware are not high. There is no doubt about the principle opportunity to build the radar equipment to detect people behind barriers. That was proven by researches published $[1,2]$ and by the authors as well [3-5].

\section{Inverse focusing problem}

The algorithm of radiotomography imaging is based on the focusing of the reflected UWB signals. The main idea of the data processing is referred to the technology of the synthetic aperture radar with the focusing. All signals, recorded at different transmitting-receiving points $\boldsymbol{\rho}$, are summed in phase for each predefined focusing point $\mathbf{r}$ with the delay correction:

$$
F(\mathbf{r}) \equiv \sum_{j} S\left(\boldsymbol{\rho}_{\mathbf{j}}, t=2 \frac{\left|\mathbf{r}-\boldsymbol{\rho}_{\mathbf{j}}\right|}{c}\right) .
$$

The transmitting receiving points can be randomly located. In approximation of the single scattering, when $S_{0}(\mathrm{t})$ is transmitted signal and the received one is

$$
S\left(\boldsymbol{\rho}_{j}, t\right)=k_{0}^{2} \iiint_{V} S_{0}\left(t-2 \frac{\left|\mathbf{r}_{1}-\boldsymbol{\rho}_{j}\right|}{c}\right) \frac{\Delta \varepsilon\left(\boldsymbol{\rho}_{1}, z_{1}\right)}{\left(4 \pi\left|\mathbf{r}_{1}-\boldsymbol{\rho}_{j}\right|\right)^{2}}\left(d^{3} \mathbf{r}_{1}\right),
$$

the following equation can be written

$$
F(\mathbf{r})=\iiint_{V} \Delta \varepsilon\left(\boldsymbol{\rho}_{1}, z_{1}\right) Q\left(\boldsymbol{\rho}_{1}, z_{1} ; \mathbf{r}\right)\left(d^{3} \mathbf{r}_{1}\right)
$$

where

$$
Q\left(\boldsymbol{\rho}_{1}, z_{1} ; \mathbf{r}\right)=\sum_{j} S_{0}\left(t=2 \frac{\left|\mathbf{r}-\boldsymbol{\rho}_{j}\right|}{c}-2 \frac{\left|\mathbf{r}_{1}-\boldsymbol{\rho}_{j}\right|}{c}\right) \frac{k_{0}^{2}}{\left(4 \pi\left|\mathbf{r}_{1}-\boldsymbol{\rho}_{j}\right|\right)^{2}}
$$

works as the hardware function of the system. With the data sufficiency, this function approximates to the «blurred» $\delta$ function which provides the identification of the focusing results with the $\Delta \varepsilon$ permittivity distribution:

$$
F(\mathbf{r}) \sim \Delta \varepsilon(\boldsymbol{\rho}, z) .
$$

As previously stated, the task of the sensing behind barriers is reduced to the detection of moving objects in some cases. Some research proposed the differential - residual technique for the detection of moving people based on filtering the signals from the stationary objects. The point of this technique is in calculation of the differential signal in successive periods of time.

$$
d S\left(t, T_{i}\right)=S\left(t, T_{i}\right)-S\left(t, T_{i-1}\right),
$$

where $d S\left(t, T_{i}\right)$ is the filtered signal, $S\left(t, T_{i}\right)$ is the recorded signal, $S\left(t, T_{i-1}\right)$ is the signal received in the previous time period. In this case, the signals reflected from the stationary background objects located in the test area are equal in different time periods and filtered by subtraction. The signal difference $d S$, obtained from the moving object is other than zero and can be used for the radar imaging the moving objects.

However, that approach didn't consider the speed of the sounded object. When the object doesn't move, the difference of signals from neighboring frames is close to zero. This results in disappearance of the information spot in the radar image. The authors proposed the modified algorithm accounting the object speed to determine the optimal sampling rate 
that maximizes the SNR for the differential signal. So, the previous equation was modified to

$$
d S\left(t, T_{i}\right)=S\left(t, T_{i}\right)-S\left(t, T_{i-N}\right),
$$

where $\mathrm{N}$ is the number of the frame that provides the maximal intensity of the differential signal.

When a person doesn't move, their presence behind the barrier can be detected by breathing movements of their chest or abdomen. Study the Figure 1 . The $S_{2}$ signal reflected from the chest during the exhalation differs from the $S_{1}$ signal reflected during the inhalation with the delay $T=2 \Delta x / c$, where $\Delta x$ is the chest shift when inhalation/ exhalation.

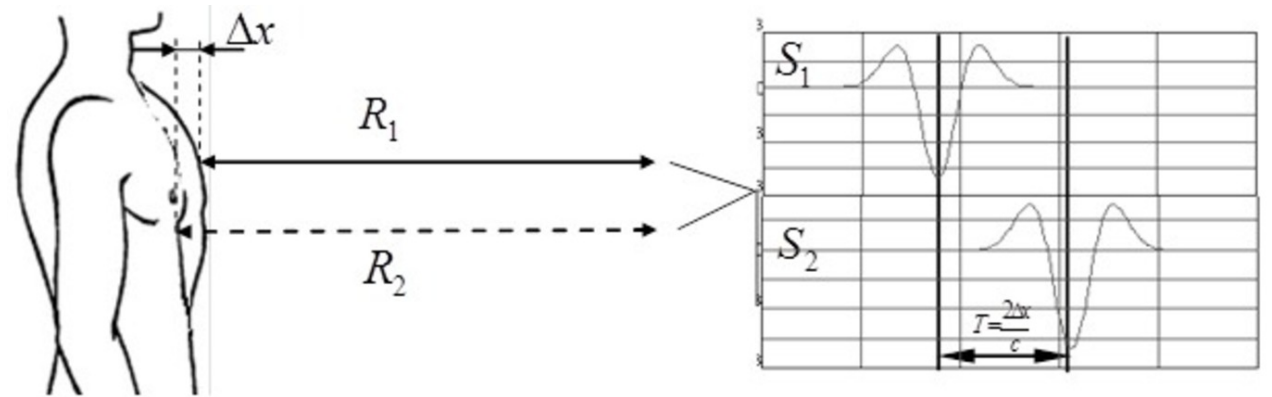

Figure 1. Record of human breath oscillation.

The data obtained in UWB sensing to be converted to distinguish the human breath oscillations. Let's consider the reflected signal spectra. The complex amplitudes of the spectrum signals received in sequential time periods agree up to the $\exp (i \omega T)$ factor:

$$
S_{2}(\omega)=S_{1}(\omega) \exp (i \omega T)
$$

When multiplying the $S S_{i}(\omega)$ spectra of the received signals by the $S_{1}(\omega)^{*}$ complex conjugated spectrum, we obtain:

$$
S_{i}(\omega) S_{1}(\omega)^{*}=\left|S_{i}\right|\left|S_{1}\right| \exp \left(i\left[\varphi_{i}-\varphi_{1}\right]\right)=\left|S_{i}\right|\left|S_{1}\right| \exp (i \omega T) .
$$

If we separate the phase from the equation obtained, then

$$
T_{i}=\frac{\arg \left(S_{i}(\omega) S_{1}(\omega)^{*}\right)}{\omega}, \omega=2 \pi f,
$$

where $f$ is the significant frequency picked out from the received signal spectrum by the predefined level.

\section{Experimental results}

To obtain multiangle projections of the sounded area, an UWB clocked array of 8 receivers and 1 transmitter was used. The receiving antennas were polled sequentially in clocking mode. The arrangement of the elements in the array is presented in Figure 2, $a$. The array element [2] is presented in Figure 2, $b$. It was a printed antenna with a screen in form of the metal angle. The developed antenna is a plate of the foil-laminated glass fiber $110 \times 110 \times 1$ $\mathrm{mm}$ in size. The arrow points the direction of maximal radiation. The antenna is designed for the radiation of UWB pulses with duration of $0.3 \ldots 0.4 \mathrm{~ns}$. 


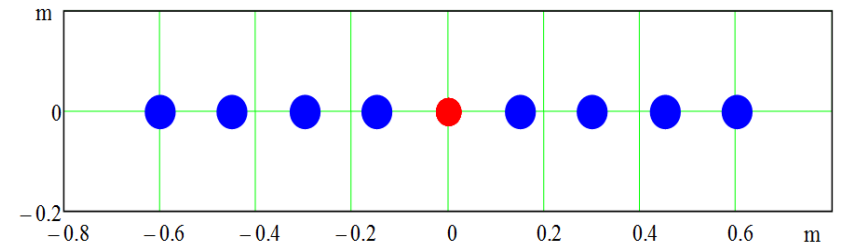

(a)

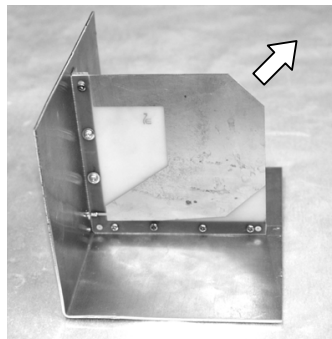

(b)

Figure 2. Arrangement of array elements (a), UWB antenna (b).

The experiment proving this approach was conducted when a person changed their location against the array. The oscilloscope record of the test area for one of the array receivers is presented in Figure 3, $a$. The signal amplitude is displayed in grayscale. The horizontal axis is the record time of the UWB signal in the stroboscopic receiver. The vertical axis is the poll time. The time between two poll periods was $0.1 \mathrm{~s}$. The Figure shows the object moving represented by changing in the arrival time of the UWB signal. The interface of the software for positioning of the moving people is presented in Figure 3, $b$. The data processing result - a tomogram of the test area in form of a $2 \mathrm{D}$ image with the cell size $50 \times 50 \mathrm{~cm}-$ is displayed in the main window.

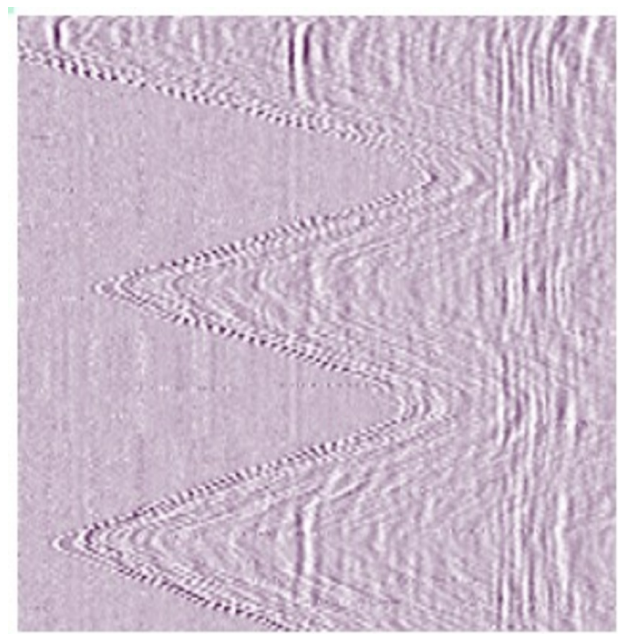

(a)

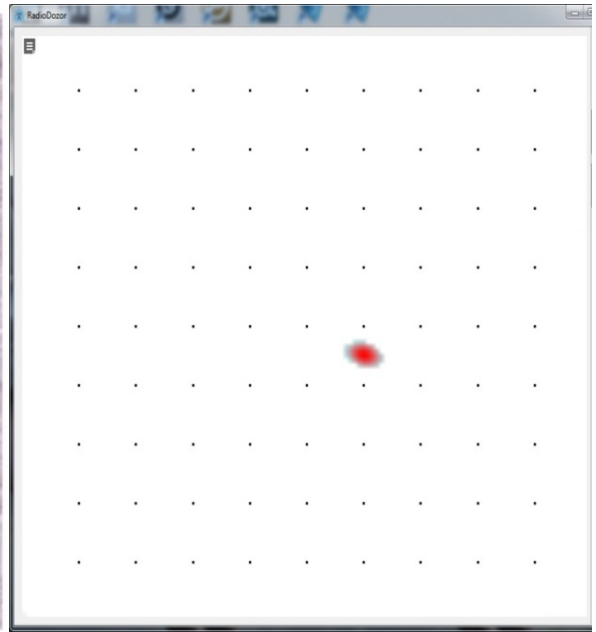

(b)

Figure 3. Oscilloscope record of the test area (a), radio image of a moving person at a certain time (b).

The proposed algorithms and applications were used for processing the results of measurement. Figure 4, $a$ presents 50 waveforms of the reflected UWB signals which were processed using the technique of the constant component filtering. The grayscale indicates the different signal levels. $\mathrm{X}$ axis corresponds to the waveform of reflected signal. Y scale is clocking time with the step of $0.5 \mathrm{~s}$. The oscillations of the human chest could be clearly found in Figure 4, $a$ as the change in clocking time of reflected signal maximum. The interface of the software for positioning by the breathing movements is presented in Figure $4, b$. The data processing result - a tomogram of the test area in form of a $2 \mathrm{D}$ image with the cell size $50 \times 50 \mathrm{~cm}-$ is displayed in the main window. 


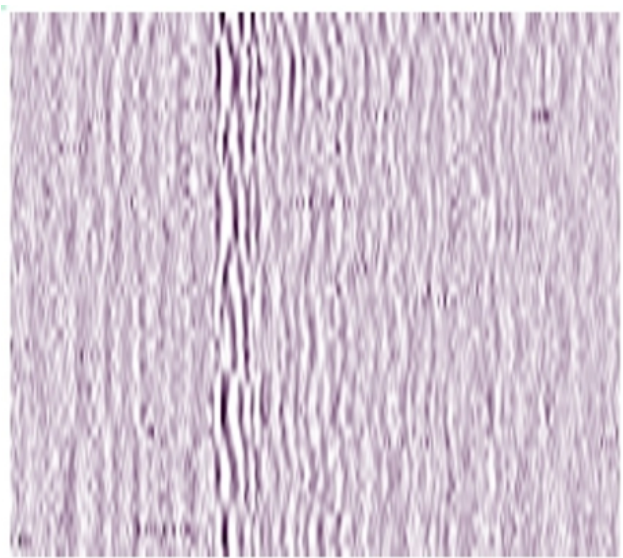

(a)

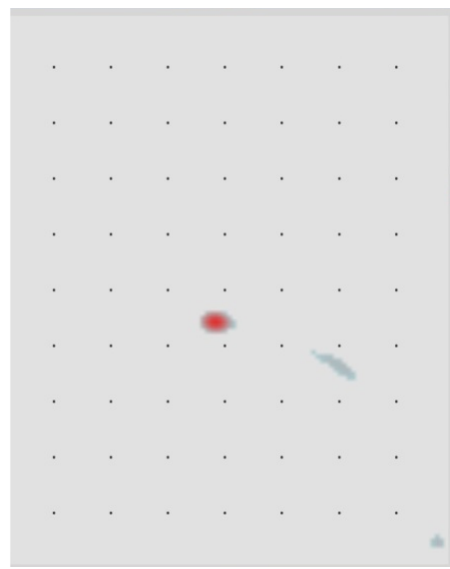

(b)

Figure 4. Oscilloscope record of test area (a), radio image of a breathing person at a certain time point (b).

As a result of oscilloscope record processing, the human chest oscillation (Figure 5, a) were distinguished. Then, using the Fourier transform, the spectrum was calculated which maximum corresponds to the breathing rate (Figure 5, $b$ ).

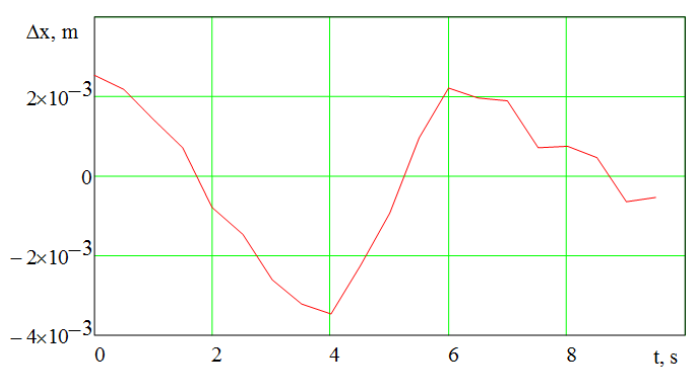

(a)

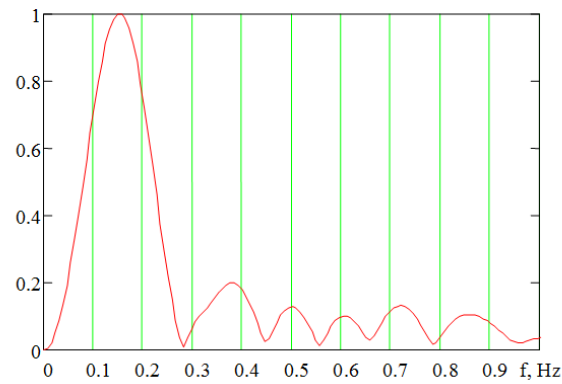

(b)

Figure 5. Waveform of experimentally distinguished oscillation of human chest (a), and its spectrum (b).

\section{Conclusion}

The research proposed the approach to detect and visualize people behind the dielectric barriers. The opportunity to obtain the radar images of moving and not moving people was demonstrated. The oscillation rate of a human chest can be defined by the continuous recording the not moving person. The research results can be used in intelligence service as well as for searching people trapped under the rubble. The range of application to be extended considering the particular conditions.

\section{Acknowledgment}

The research was supported by the Russian Ministry of Education and Science as a part of the state order No 3.694.2014/K. 


\section{References}

[1] Yazhou Wang, Aly E. Fathly, IEEE Trans. on Geoscience and Remote Sensing 50, 1986 (2012) doi: 10.1109/TGRS.2011.2170694

[2] R.N. Satarov, Yu. Kuz'menko, T. R. Muksunov, A. V. Klokov, E. V. Balzovskii, Yu. I. Buyanov, S. E. Shipilov, V. P. Yakubov, Russian Physics Journal 55, 884 (2013) doi: 10.1007/s11182-013-9896-8

[3] James D. Taylor, Ultra wideband radar. Applications and design (Crc Press, NewYork, 2012)

[4] Jurgen Sachs, Handbook of ultra-wideband short-range (Wiley-vch, 2012)

[5] Y. Kan, Y. Zhu, Q. Fu, MATEC Web of Conferences 56, 02001 (2016) doi: $10.1051 /$ matecconf $/ 20165602001$ 